\title{
Cost Pattern Comparison between Survivor-and Non- survivor of Mechanically-Ventilated COVID-19 Patients
}

\author{
Antonius Abimasetyo Putro ${ }^{\mathrm{a}}$, K. Kuntaman ${ }^{\mathrm{b}, \mathrm{d}}$, Bambang Pujo Semedi $^{\mathrm{c}, \mathrm{d}^{*}}$ \\ ${ }^{a}$ Study Program of Specialist in Anaesthesiology and Reanimation, Department of Anaesthesiology and \\ Reanimation, Faculty of Medicine Universitas Airlangga \\ ${ }^{b}$ Department of Medical Microbiology, Faculty of Medicine Universitas Airlangga \\ 'Department of Anaesthesiology and Reanimation, Faculty of Medicine Universitas Airlangga \\ ${ }^{\mathrm{d} D r}$. Soetomo General Academic Hospital, Surabaya, Indonesia
}

Corresponding author: Bambang Pujo Semedi, email: bpsanest@gmail.com

\begin{abstract}
This study aimed to identify the cost analysis between survivor and non-survivor of mechanically ventilated COVID-19 patients. We conducted a cohort retrospective study analysing the cost comparison among mechanically ventilated COVID-19 patients in our hospital, DR.Soetomo Hospital, which is a tertiary referral hospital in our country, from from June to September 2020. COVID-19 patients who was mechanically ventilated was included in our study, but for those who were passed away $<24$ hours upon ICU admission, or patients who were discharge against medical advice, were excluded from this study. A total of 72 mechanically ventilated COVID-19 patients were analysed. These patients were divided into 2 groups: survivor and non-survivor groups. Survivor group consisted of 21 patients and 51 patients in non-survivor group. Baseline characteristics were significantly different for the variables of: body mass index, presenting blood pressure, acute kidney injury complication, prothrombin time, albumin, P/F ratio and SOFA scores. The average total cost spent in survivor group was $\$ 3711,7$ while in non-survivor group was $\$ 5417,7$. This result showed a significant finding ( $\mathrm{p}=0.047)$. Cost distribution pattern was similar in both groups, that cost medical items was the highest cost that spent during ICU stays among mechanically-ventilated COVID-19 patients; but significant difference of medical item cost was documented between survivor and non-survivor group. An average of cost medical items was spent of $\$ 1145,5$ in survivor group, compared to $\$ 2353,8$ in non-survivor group ( $\mathrm{p}=0.003$ ). We conclude that non-survivor group of mechanically ventilated patients tend to spend more cost during ICU stays, especially for the medical items cost.
\end{abstract}

Keywords: Hospital Cost, COVID-19, Mechanical Ventilator, Discharge outcome

\section{Introduction}

World Health Organization (WHO) has established COVID-19 as a Public Health Emergency of International Concern (PHEIC) since $30^{\text {th }}$ of January 2020, and has declared it as a pandemic on March $11^{\text {th }}, 2020$. COVID-19 is caused by Severe Acute Respiratory Syndrome coronavirus 2 (SARS-CoV-2), which formerly was called 2019-nCoV.[1] This RNA virus caused a various clinical manifestations. Some COVID-19 patients were asymptomatic, some other felt dyspnoea, and there were patients with severe complications, like sepsis, acute respiratory distress syndrome (ARDS), and multiorgan failure. Special care should be given to the elderly, immunocompromised, or to the pregnant patients with COVID-19. To date, no curative treatment has been established and approved as the treatment guideline standard to all COVID-19 patients around the world.[2]

COVID-19 pandemic caused a marked increase in healthcare expenditures and critical availability of hospital resources, such as hospital beds, intensive care, ventilators, medications, etc. Patient characteristics and ventilation practices were evolving over time, and improvements of mechanically-ventilated patients was improving.[3] The scant availability of ventilator for severe and critical COVID-19 patients needed special attention because intensive care required relatively expensive costs which become one of the high expenditures of the hospitals. Thus, description and analysis of therapeutical and healthcare system expenditure become an important aspect to be studied further in mechanically ventilated COVID-19 patients. Expenditure calculation and its correlation with components of healthcare service could be analysed.

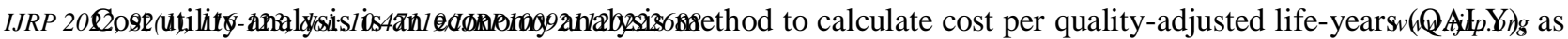
the cost differences between 2 interventions divided by the QALY differences that were given from those 2 
interventions. Cost utility analysis was developed to overcome cost-effectivity issues and could help decision making to compare different value from each intervention in various health problems. Utility value could be achieved by using standardized and validated health status instruments, so that the evidence of cost utility analysis could be directly applied with an affordable cost.[4]

This study aimed to investigate the different pattern of hospitalization expenditure between survivor and non-survivor of mechanically ventilated COVID-19 patients. This study would provide a recent national reference to the allocation of the intensive care resources to those most in need. Hopefully, this study will give a substantial benefit as the basis cost analysis for the governments and healthcare insurance to make efficient money spending and effective decisions that helps both the patients that really need the mechanical ventilation in this time of crisis.

\section{Methods}

\subsection{Study Design}

This was an observational retrospective study using medical records data based on the case record forms in confirmed COVID-19 patients who were mechanically ventilated in RSUD dr. Soetomo COVID-19 ICU, from June to September 2020. This study investigated the effectivity of healthcare expenditure towards the clinical outcomes of mechanically ventilated COVID-patients in our Centre, as well as the different hospital expenditure patterns toward the patients' clinical outcome at ICU discharge (survivor compared to non-survivor).

\subsection{Study population}

This study used total sampling. So that the study participants were all of the COVID-19 patients who were mechanically ventilated in the period from June to September and did not met one of the following criteria: (1) patients with ICU care of $\leq 48$ hours, (2) patients who discharged against medical advice (DAMA). All of the eligible patients were followed-up until ICU discharge. Patients' discharge outcomes were documented and the data were analysed and grouped based on the patients' discharge outcomes (survivor compared to non-survivor).

\subsection{Measurements}

Indications of mechanically ventilator use in COVID-19 patients in our centre were as the following:[5]

- Airway problems

- Progressive deterioration of dyspnoea accompanied with the presence of: altered mental status, respiratory rate $>30$ times per minutes. $\mathrm{SpO} 2<90 \%$, or the presence of shock.

- Hypoxemic patients with $\mathrm{SpO} 2<93 \%$ ( $\mathrm{FiO} 221 \%$ ), $\mathrm{PaO} 2 / \mathrm{FiO} 2<300 \mathrm{mmHg}$ with progressive worsening or with the increasing of additional respiratory muscle or with pulse of $>120$ times per minute or with the ROX index $<3.85$ (in HFNC)

\subsection{Variables and outcome}

Patients' ICU discharge outcome became the dependent variables, and hospital total cost and hospital costs categories became the independent variables.(Table 1)

Table 1 Categories of Hospital cost and their components

\begin{tabular}{|l|l|}
\hline \multicolumn{1}{|c|}{ Healthcare expenditure } & \multicolumn{1}{c|}{ Components } \\
\hline Cost Hospital Service & $\begin{array}{l}\text { COVID-19 ICU, Ventilator, High Flow Nasal Canule (HFNC), Continuous Renal } \\
\text { Replacement Therapy (CRRT), Haemodialysis, Syringe pump, Infusion pump, Monitor, } \\
\text { Extracorporeal Membrane Oxygenation (ECMO), Oksigen. }\end{array}$ \\
\hline Cost Medical Service & Nursing care service, doctors' visit, consultation service. \\
\hline Cost Medical Item & $\begin{array}{l}\text { medications (enteral dan parenteral), disposable items (infusion set, syringes, electrodes, } \\
\text { urine catheters, CVC, sanitary napkins, suction, plaster, BGA cartridge, mucous } \\
\text { extractor, needles, personal protective equipment). }\end{array}$ \\
\hline Cost Diagnostic Service & $\begin{array}{l}\text { Radiographic service, clinical pathology and microbiology service, blood bank } \\
\text { installation service }\end{array}$ \\
\hline Cost Medical Therapy & $\begin{array}{l}\text { Intubation, Central Venous Catheter (CVC) insertion, appliance of Extracorporeal } \\
\text { Membrane Oxygenation (ECMO), installation of Continuous Renal Replacement } \\
\text { Therapy (CRRT), insertion of Invasive Blood Pressure (IBP), other surgical or invasive } \\
\text { treatments. }\end{array}$ \\
\hline
\end{tabular}




\subsection{Statistical analysis}

Descriptive statistics analysed the baseline characteristics of mechanically-ventilated COVID-19 patients. Statistical analysis that compared hospital expenditure categories between survivor and non-survivor was using a comparison test. Kolmogorov-Smirnov was used for normality test of hospital expenditures, with $\mathrm{p}<0,05$ was considered to be heterogenous, thus the non-parametric comparison test was used, Mann Whitney test.

\section{Results}

\subsection{Baseline characteristics}

According to the inclusion and exclusion criteria, a total of 72 mechanically ventilated COVID-19 patients were analysed. These patients were divided into 2 groups: survivor and non-survivor groups. Survivor group consisted of 21 patients and 51 patients in non-survivor group. The average of age was 47 years old in both groups. Male patients were more prominent in non-survivor group, but not significantly different. Body mass index (BMI) in survivor group was $25 \mathrm{~kg} / \mathrm{m}^{2}$ in the average, but non-survivor group had an average BMI of $29 \mathrm{~kg} / \mathrm{m}^{2}$, which were significantly different. Length of stay was not significantly different between survivor and non-survivor group, with the average of 9 days. The presenting vital signs upon ICU admission were significantly different between two groups. Systolic blood pressure, diastolic blood pressure and mean arterial pressure were significantly lower in the non-survivor group, but body temperature did not differ both groups.

Some laboratory results among COVID-19 patients who were mechanically ventilated were significantly different between survivor and non-survivor group. In survivor group, Blood Urea Nitrogen was lower, albumin was higher, and prothrombin time was shorter in duration compared to the non-survivor group. The other laboratory results showed no significant findings. Complication of Acute kidney injury was found mostly in the non-survivor group, but no comorbidities signify the difference between both groups. The highest SOFA score during ICU stays significantly higher in the non-survivor group, which was 13 points, compared to 7 points in the survivor group.

Antivirals, antifungals, and antibiotics were given properly as indicated. Antibiotics consumption was higher in non-survivor group, but anti-viral and anti-fungal were equally administered among the mechanically ventilated COVID-19 patients. (Table 2)

Table 2. Baseline characteristics of survivor and non-survivor in mechanically-ventilated COVID-19 patients

\begin{tabular}{|c|c|c|c|c|}
\hline Variables* & $\begin{array}{l}\text { All patients } \\
\qquad \mathrm{N}=72\end{array}$ & $\begin{array}{c}\text { Survivor } \\
\mathrm{n}=21(29.1 \%)\end{array}$ & $\begin{array}{l}\text { Non-Survivor } \\
\mathrm{N}=51(70.8 \%)\end{array}$ & P-value \\
\hline Age & $47.7 \pm 12.7$ & $47.5 \pm 11.4$ & $47.7 \pm 13.2$ & 0.958 \\
\hline Male Gender & $45(62.5 \%)$ & $10(47.6 \%)$ & $35(68.6 \%)$ & 0.114 \\
\hline Body Mass Index & $28.3 \pm 6.2$ & $25.2 \pm 4.3$ & $29.6 \pm 6.4$ & $0.006^{*}$ \\
\hline Length of stay (days) & $9.3 \pm 5.1$ & $8.9 \pm 4.2$ & $9.5 \pm 5.5$ & 0.628 \\
\hline \multicolumn{5}{|l|}{ Vital signs } \\
\hline Systolic Blood Pressure (mmHg) & $91.2 \pm 19.8$ & $104.3 \pm 16.0$ & $85.7 \pm 18.6$ & $<0.001 *$ \\
\hline Diastolic Blood Pressure(mmHg) & $50.8 \pm 12.3$ & $56.7 \pm 11.9$ & $48.3 \pm 11.8$ & $0.008^{*}$ \\
\hline Mean Arterial Pressure & $63.2 \pm 14.1$ & $71.7 \pm 12.2$ & $59.8 \pm 13.3$ & $0.001 *$ \\
\hline Body Temperature & $37.5 \pm 4.1$ & $37.6 \pm 0.3$ & $37.5 \pm 4.8$ & 0.925 \\
\hline \multicolumn{5}{|l|}{ Laboratory results } \\
\hline Haemoglobin & $11.1 \pm 1.9$ & $10.8 \pm 1.7$ & $11.1 \pm 2.1$ & 0.612 \\
\hline Haematocrit & $33.4 \pm 6.0$ & $32.4 \pm 5.1$ & $33.8 \pm 6.3$ & 0.358 \\
\hline Thrombocyte & $326173 \pm 31796$ & $346857 \pm 16114$ & $317656 \pm 36459$ & 0.726 \\
\hline Leucocyte & $23511 \pm 11629$ & $23332 \pm 12358$ & $23584 \pm 11442$ & 0.934 \\
\hline Lactate & $2.5 \pm 3.4$ & $1.6 \pm 0.8$ & $2.8 \pm 3.9$ & 0.128 \\
\hline SGOT & $99.4 \pm 138.3$ & $65.2 \pm 42.2$ & $87.6 \pm 63.3$ & 0.175 \\
\hline SGPT & $82.7 \pm 60.5$ & $70.71 \pm 52.6$ & $87.6 \pm 63.3$ & 0.285 \\
\hline Blood Urea Nitrogen & $59.9 \pm 49.01$ & $40 \pm 37.9$ & $68.1318 .0 \pm 50.9$ & $0.026^{*}$ \\
\hline Creatinine Serum & $3.8 \pm 5.9$ & $2.6 \pm 5.4$ & $5.4 \pm 6.0$ & 0.269 \\
\hline C-Reactive Protein & $17.0 \pm 37.5$ & $11.9 \pm 38.9$ & $6.4 \pm 9.3$ & 0.429 \\
\hline Procalcitonin & $10.8 \pm 2.5$ & $2.3 \pm 3.6$ & $14.3 \pm 30.1$ & 0.074 \\
\hline Blood sugar & $289 \pm 132.2$ & $282.8 \pm 131.6$ & $292.8 \pm 133.5$ & 0.773 \\
\hline HBA1C & $6.9 \pm 2.2$ & $6.7 \pm 2.2$ & $7.0 \pm 1.9$ & 0.586 \\
\hline
\end{tabular}




\begin{tabular}{|c|c|c|c|c|}
\hline D-dimer & $6478 \pm 8137$ & $6148 \pm 7651$ & $6614 \pm 8398$ & 0.827 \\
\hline Ferritin & $1506 \pm 2361$ & $950.1 \pm 705.6$ & $1735 \pm 2745$ & 0.202 \\
\hline PTT & $14.4 \pm 4.1$ & $12.6 \pm 2.0$ & $15.2 \pm 4.5$ & $0.002^{*}$ \\
\hline APTT & $48.6 \pm 30.7$ & $31.5 \pm 18.8$ & $55.6 \pm 33.5$ & 0.675 \\
\hline Albumin & $2.8 \pm 0.3$ & $2.8 \pm 0.3$ & $2.7 \pm 0.2$ & $<0.001 *$ \\
\hline \multicolumn{5}{|l|}{ Respiratory } \\
\hline $\mathrm{P} / \mathrm{F}$ ratio & $94.0 \pm 64.8$ & $150.5 \pm 89.3$ & $86.9 \pm 30.0$ & $<0.001 *$ \\
\hline $\mathrm{SPO}_{2}$ & $94.0 \pm 5.1$ & $93.9 \pm 4.0$ & $91.8 \pm 4.5$ & 0.059 \\
\hline SOFA score & $12 \pm 4.3$ & $7 \pm 4.3$ & $13.3 \pm 3.1$ & $<0.001^{*}$ \\
\hline High Flow Nasal Cannula & $48(39 \%)$ & $6(28.5 \%)$ & $12(23.5 \%)$ & 0.653 \\
\hline \multicolumn{5}{|l|}{ Comorbidities } \\
\hline Hypertension & $31(43.1 \%)$ & $9(42.9 \%)$ & $18(32.1 \%)$ & 1.000 \\
\hline Diabetes Mellitus & $37(51.4 \%)$ & $7(33.3 \%)$ & $30(60.0 \%)$ & 0.067 \\
\hline Chronic Kidney Disease & $1(1.4 \%)$ & $0(0.0 \%)$ & $1(1.9 \%)$ & 1.000 \\
\hline Asthma & $2(2.8 \%)$ & $0(0.0 \%)$ & $2(3.9 \%)$ & 0.581 \\
\hline Hepatitis & $13(18.1 \%)$ & $6(28.6 \%)$ & $7(13.7 \%)$ & 0.180 \\
\hline Acute Kidney Disease & $35(48.6 \%)$ & $6(28.6 \%)$ & $20(40 \%)$ & $0.039^{*}$ \\
\hline \multicolumn{5}{|l|}{ Medications } \\
\hline Dopamine & $2(2.8 \%)$ & $1(4.8 \%)$ & $1(1.9 \%)$ & 0.501 \\
\hline Dobutamine & $20(27.8 \%)$ & $5(23.8 \%)$ & $15(29.4 \%)$ & 0.775 \\
\hline Norepinephrine & $48(66.7 \%)$ & $8(38.0 \%)$ & $40(78.4 \%)$ & $0.002 *$ \\
\hline Epinephrine & $25(34.7 \%)$ & $1(4.7 \%)$ & $24(47.1 \%)$ & $0.001 *$ \\
\hline Vasopressin & $21(29.2 \%)$ & $1(4.8 \%)$ & $20(39.2 \%)$ & $0.003^{*}$ \\
\hline Antibiotics & $67(93.1 \%)$ & $17(80.9 \%)$ & $50(98.0 \%)$ & $0.023^{*}$ \\
\hline Antiviral & $47(65.3 \%)$ & $13(61.9 \%)$ & $34(66.6 \%)$ & 0.787 \\
\hline Antifungal & $15(20.8 \%)$ & $2(9.5 \%)$ & $25(25.5 \%)$ & 0.203 \\
\hline
\end{tabular}

\subsection{Cost comparison among mechanically-ventilated COVID-19 patients}

The average total cost spent in survivor group was $\$ 3711,7$ while in non-survivor group was $\$ 5417,7$. This result showed a significant finding $(\mathrm{p}=0.047)$. Cost distribution pattern was similar in both groups, that cost medical items was the highest cost that spent during ICU stays among mechanically-ventilated COVID-19 patients; but significant difference of medical item cost was documented between survivor and non-survivor group. An average of cost medical items was spent of $\$ 1145,5$ in survivor group, compared to $\$ 2353,8$ in non-survivor group $(\mathrm{p}=0.003)$. 


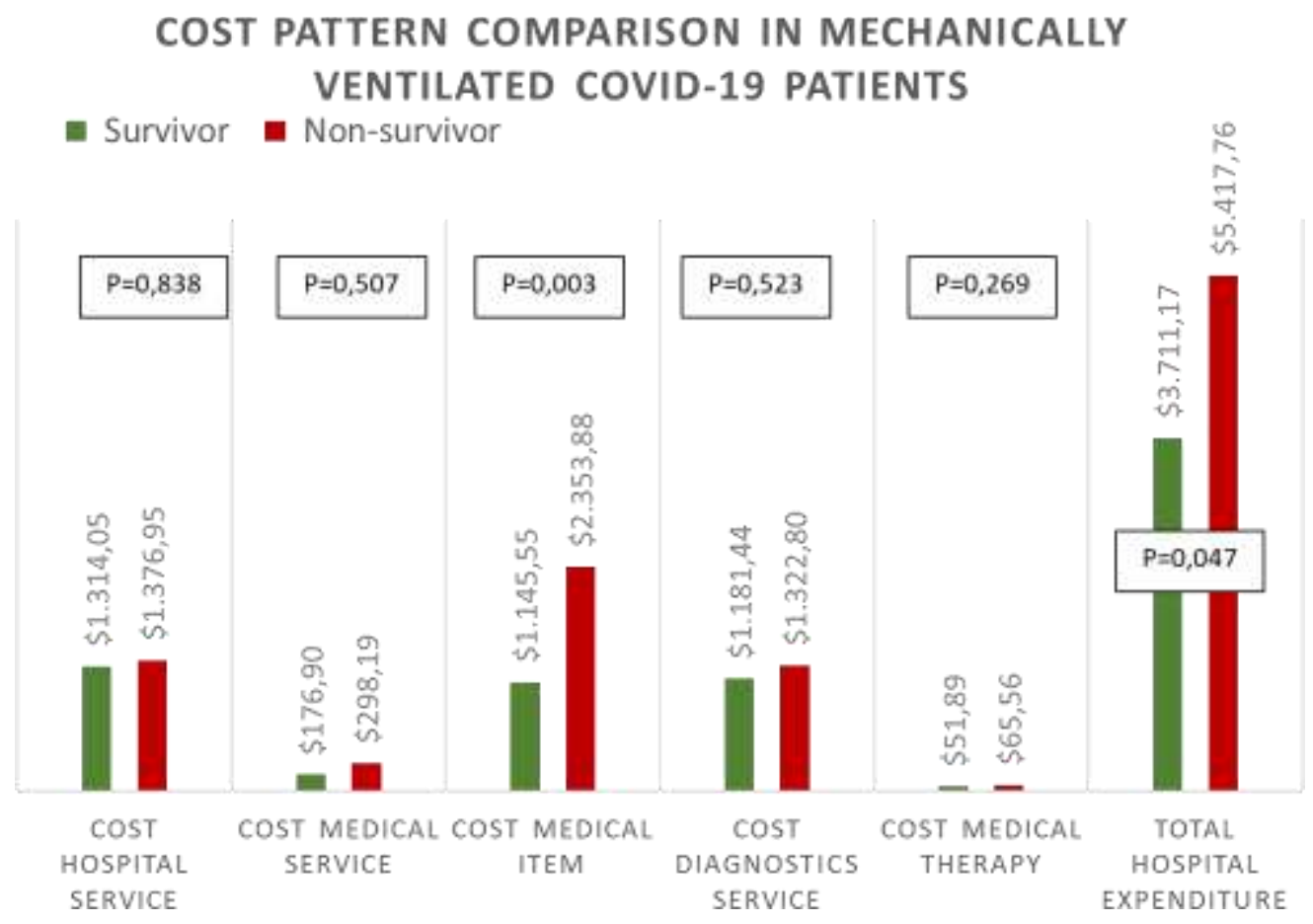

Fig. 1. Cost Pattern Comparison in Survivor and Non-Survivor of Mechanically-Ventilated COVID-19 patients.

\section{Discussion}

Mechanically-ventilated COVID-19 patients were more profound in the average age of 47 which was the productive age. This result was alongside with observation from other hospital in Jakarta, with median age of 46 years old.[6] Prior study investigated that SARS-COV-2 incubation period of younger age population was longer than the elderly.[7] In Indonesia, patients with productive age dominated the COVID-19 incidence, because this population was actively working and prone to many physical contacts. Male patient was more prominent in mechanically-ventilated COVID-19 patients, but not significantly different. Generally, in Indonesia the number of male patients were indeed dominant among the COVID-19 patients.[6]

Physiologic and immunologic differences in male and female influenced the host response to infections, including SARS-COV-2 infection.[8] Female likelihood to restrain COVID-19 infection was correlated to the protective effect of $\mathrm{X}$ chromosome and oestrogen that were beneficial for adaptive immunity.[9]

Most of the patients in our study had length of stay of 9 days in both groups. Those patients were discharged from the COVDI-19 ICU either moved to low-care units, passed away, or transferred to non-COVID ICU. On the contrary, a prior study in Wuhan which included 1792 patients showed that the average length of stay in ICU was 11 days, with the range of 6 to 20 days. Length of stay of those patients was correlated with their ages.[10] Another study from Hongkong stated that the average length of stay in ICU was 16 days.[11] These differences could be because of the different characteristics of the patients, for example: age and comorbidities that also could influence the disease severity, and subsequently the length of stay in ICU. Some laboratory results also had strong correlation with the length of stay in ICU, for example the time of thromboplastin activation, leukocyte count, and albumin levels. These laboratory results described the patients' conditions and the disease progression.[11]

The most prevalent comorbidity in mechanically-ventilated COVID-19 patients were Diabetes mellitus in $51 \%$ of the patients, followed by hypertension in $31 \%$ of the patients. These results were in accordance with a prior study that explored the 3 most common comorbidities found in severe and critical COVID-19 patients, they were hypertension $(52,1 \%)$, diabetes $(33,6 \%)$, dan and the other cardiovascular disease $(20,9 \%)$. These comorbidities highly influenced the number of hospitalised patients, length of stay, as well as mortality, if compared to the patients without comorbidity.[9]

Mechanical ventilation was indicated for patients with a progressive deterioration of dyspnoea accompanied with the presence of: altered mental status, respiratory rate $>30$ times per minutes. SpO2<90\%, or the presence of shock. 
It also recommended to be used for Hypoxemic patients with $\mathrm{SpO} 2<93 \%$ (FiO2 21\%), PaO2/FiO2 <300mmHg with progressive worsening or with the increasing of additional respiratory muscle or with pulse of $>120$ times per minute or with the ROX index <3.85 (in HFNC).[5]

Cost analysis in our study was done in survivor and non-survivor groups. There were 5 categories of hospital expenditure that were observed, they were: Cost Hospital Service, Cost Medical Service, Cost Medical Item, Cost Diagnostic Service, Cost Medical Therapy. Among these expenditure categories, median cost of medical therapy was the lowest cost that should be paid by the patients, followed by cost medical service. In survivor group, the highest expenditure was spent by the category of cost hospital service. Whereas in non-survivor group, cost medical item consumed the most of the expenditure, continued with cost hospital service and cost diagnostic service, and subsequently cost medical service and cost medical therapy.

Cost Hospital Service consisted of the cost of isolation room specific for COVID-19, medical device installation like ventilator, High Flow Nasal Canula (HFNC), Continuous Renal Replacement Therapy (CRRT), Haemodialysis, syringe pump, infusion pump, monitor, Extracorporeal Membrane Oxygenation (ECMO), oxygen consumption, administration cost, and admission cost. The average of hospital service cost in survivor group was \$1314 and \$1376 in non-survivor group. Renal Replacement Therapy (RRT) was needed to dispose excess fluid in bloodstream, as well as switching solutes like toxins but also essential substances like potassium, calcium, chloride, bicarbonate of body fluid with the dialysate. Generally, there were 3 types of RRT: (1) Intermittent Haemodialysis (IHD), (2) Peritoneal Dialysis (PD) (3) Continuous Renal Replacement Therapy (CRRT).[12] AKI was commonly seen in severe and critical COVID-19 patients who were taken care in ICU RSUD Dr. Soetomo Surabaya. This could be acquired because the severe manifestation that called sepsis. Patients with AKI complication needed the CRRT to filtrate continuously for 24 hours, so that it could maintain the haemodynamic of the patients. CRRT was not found in every centre in Indonesia, and the price of CRRT instalment was relatively expensive. In our centre, CRRT cost for Rp 13.950.000, - (\$962) for each day, consisted of facility cost of Rp 10.089.000,- (\$695) and service cost of Rp 3.861.000, -(\$266,3). Extracorporeal Life Support (ECLS) aimed to return the impaired respiratory function by maintaining oxygenation and adequately eliminating $\mathrm{CO}_{2}$. Some types of ECLS were: (1) Extracorporeal Lung Axis (ECLA), (2) Extracorporeal Membrane Oxygenation (ECMO), (3) Extracorporeal Carbon Dioxide Removal (ECOO2R), and (4) Extracorporeal Cardiopulmonary Resuscitation (ECPR).[13] ECMO use was increased in year 2009 and 2010 for the management of porcine flu (due to H1N1 virus).[14] Nowadays, ECMO utilization also helps COVID-19 patients with severe cardiopulmonary problems in which no conventional therapy could help. Working principle of ECMO is like Cardiopulmonary Bypass (CPB), so practical that allows the exchange of gases outside the body in a longer duration. ECMO utilization in our centre cost for $\mathrm{Rp} 70.832 .350$,-(\$18678) on the first day and service cost for Rp 20.790.000,-(\$1434), it cost Rp 7.822.000,-(\$539) for the facility cost and Rp 3.861.000,-(\$266) for the service cost on the next days.

Cost Diagnostic Service consisted of radiographic service, clinical pathology service, clinical microbiology service, blood bank service of blood type test and blood packs reservation. An average cost of $\$ 1181$ in survivor group compared to $\$ 1322$ in non-survivor group of mechanically ventilated patients. Cost Medical Therapy consisted of invasive treatments like intubation, insertion of Central Venous Catheter (CVC), utilization of ECMO, utilization of CRRT, instalment of Arterial Blood Pressure (ABP) and surgical procedures as indicated. Average cost of medical therapy of survivor group was $\$ 51,89$ and the average of $\$ 65.56$ was spent in nonsurvivor group of mechanically ventilated COVID-19 patients.

Cost Medical Item consisted of patients' medications, disposable items (infusion set, syringes, electrodes, urine catheter, CVC, suction, ABP, set ventilator, cartridge BGA, mucous extractor, and protective medical equipment). Mechanical ventilation had a big contribution to the hospital cost. A study from Saudi Arabia demonstrated a significant high cost expensed from the mechanically ventilated COVID-19 patients compared to those who were not mechanically-ventilated (\$2990.76 vs \$2082.65, respectively).[15] Our study showed a lower average of direct medical cost among the mechanically ventilated patients, they were $\$ 1145,5$ in survivor group and $\$ 2353,8$ in non-survivor group ( $\mathrm{p}=0.003$ ). A study from Cleary et al (2021)[16] demonstrated that shorter length of stay in ICU could effectively improve hospital expenditure, moreover, lower service cost increase daily ICU cost effectivity. Avoiding years of life lost makes ICU more cost-effective. On the contrary, in mechanicallyventilated COVID-19 patients taken care in ICU, the average of the length of stay was not significantly different between the survivor compared to the non-survivor. In mechanically ventilated COVID-19 patients, patients' discharge outcome significantly associated with the total expense, especially for the medical item cost. 


\section{Conclusion}

There was a significantly different pattern of expenditure categories that was spent by the survivors compared to the non-survivor of severe and critical COVID-19 patients who were admitted to ICU. The highest expenditure that spent by the survivor group was the diagnostic cost, while non-survivor group spend most of the expenditure for medical item. Cost medical therapy was the lowest expenditure category spent both in survivor and non-survivor group. Cost medical item, cost hospital service, cost medical service became the most important factors that influenced the total hospital expenditure in ICU and were correlated with the ICU discharge outcome. Non-survivor group spent more cost in overall expenditure category.

\section{Conflict of Interest}

The authors declared no potential conflict of interest relevant to this article.

\section{Authors' Agreement}

All authors gave final approval and agree to be accountable for all aspects of work, ensuring integrity and accuracy.

\section{REFERENCES}

1 Chavez S, Long B, Koyfman A, et al. Coronavirus Disease (COVID-19): A primer for emergency physicians. Am J Emerg Med 2021;44:220-9. doi:https://doi.org/10.1016/j.ajem.2020.03.036

2 Dhont S, Derom E, Van Braeckel E, et al. The pathophysiology of 'happy' hypoxemia in COVID-19. Respir Res 2020;21:198. doi:10.1186/s12931-020-01462-5

3 Esteban A, Frutos-Vivar F, Muriel A, et al. Evolution of mortality over time in patients receiving mechanical ventilation. Am J Respir Crit Care Med 2013;188:220-30. doi:10.1164/rccm.201212-2169OC

4 Adibe MO, Aguwa CN, Ukwe C V. Cost-Utility Analysis of Pharmaceutical Care Intervention Versus Usual Care in Management of Nigerian Patients with Type 2 Diabetes. Value Heal Reg Issues 2013;2:189-98. doi:https://doi.org/10.1016/j.vhri.2013.06.009

5 PDPI, PERKI, PAPDI, et al. Pedoman tatalaksana COVID-19. Pedoman Tatalaksana COVID-19 2020;:1-138.https://www.papdi.or.id/download/983-pedoman-tatalaksana-covid-19-edisi-3-desember2020 (accessed 10 Nov 2021).

6 Surendra H, Elyazar IR, Djaafara BA, et al. Clinical characteristics and mortality associated with COVID19 in Jakarta, Indonesia: A hospital-based retrospective cohort study. Lancet Reg Heal - West Pacific 2021;9:100108. doi:10.1016/j.lanwpc.2021.100108

7 Dai J, Yang L, Zhao J. Probable longer incubation period for elderly covid-19 cases: Analysis of 180 contact tracing data in hubei province, china. Risk Manag Healthc Policy 2020;13:1111-7. doi:10.2147/RMHP.S257907

8 Biswas M, Rahaman S, Biswas TK, et al. Association of Sex, Age, and Comorbidities with Mortality in COVID-19 Patients: A Systematic Review and Meta-Analysis. Intervirology 2021;64:36-47. doi:10.1159/000512592

9 Karyono DR, Wicaksana AL. Current prevalence, characteristics, and comorbidities of patients with COVID-19 in Indonesia. J Community Empower Heal 2020;3:77. doi:10.22146/jcoemph.57325

10 Zheng J, Zhou R, Chen F, et al. Incidence, clinical course and risk factor for recurrent pcr positivity in discharged covid-19 patients in guangzhou, china: A prospective cohort study. PLoS Negl Trop Dis 2020;14:1-14. doi:10.1371/journal.pntd.0008648

11 Zhou J, Tse G, Lee S, et al. Identifying main and interaction effects of risk factors to predict intensive care admission in patients hospitalized with COVID-19: A retrospective cohort study in Hong Kong. medRxiv Published Online First: 2020. doi:10.1101/2020.06.30.20143651

12 Tandukar S, Palevsky PM. Continuous Renal Replacement Therapy: Who, When, Why, and How. Chest 2019;155:626-38. doi:10.1016/j.chest.2018.09.004

13 Chauhan S, Subin S. Extracorporeal membrane oxygenation, an anesthesiologist's perspective: Physiology and principles. Part 1. Ann Card Anaesth 2011;14:218-29. doi:10.4103/0971-9784.84030

14 ECMO for Severe Acute Respiratory Distress Syndrome. N Engl J Med 2018;379:1090-3. doi:10.1056/NEJMc1808731

15 Khan AA, AlRuthia Y, Balkhi B, et al. Survival and Estimation of Direct Medical Costs of Hospitalized COVID-19 Patients in the Kingdom of Saudi Arabia. Int. J. Environ. Res. Public Heal. . 2020;17. doi:10.3390/ijerph17207458 
16 Cleary SM, Wilkinson T, Tamandjou Tchuem CR, et al. Cost-effectiveness of intensive care for hospitalized COVID-19 patients: experience from South Africa. BMC Health Serv Res 2021;21:82. doi:10.1186/s12913-021-06081-4 